OPEN

SUBJECT AREAS:

INNATE IMMUNITY

ANTIMICROBIALS

BACTERIA

MEDICAL RESEARCH

Received

14 March 2011

Accepted

07 April 2011

Published

14 June 2011

Correspondence and requests for materials should be addressed to

S.M.J.F. (fleiszig@ berkeley.edu)

\title{
Modulation of epithelial immunity by mucosal fluid
}

\author{
James J. Mun ${ }^{1,2}$, Connie Tam ${ }^{1}$, David J. Evans ${ }^{1,4} \&$ Suzanne M. J. Fleiszig ${ }^{1,2,3}$
}

\begin{abstract}
${ }^{1}$ School of Optometry, University of California, Berkeley, CA 94720, USA, ${ }^{2}$ Vision Science Program, University of California, Berkeley, CA 94720, USA, ${ }^{3}$ Graduate Groups in Microbiology and Infectious Disease, University of California, Berkeley, CA 94720, USA, ${ }^{4}$ College of Pharmacy, Touro University-California, Vallejo, CA 94592, USA.
\end{abstract}

\begin{abstract}
Mucosal epithelial cells, including those at the ocular surface, resist infection by most microbes in vivo but can be susceptible to microbial virulence in vitro. While fluids bathing mucosal surfaces (e.g. tears) contain antimicrobials, potentially pathogenic microbes often thrive in these fluids, suggesting that additional mechanisms mediate epithelial resistance in vivo. Here, tear fluid acted directly upon epithelial cells to enhance their resistance to bacterial invasion and cytotoxicity. Resistance correlated with tear fluid-magnified activation of NFKB and AP-1 transcription factors in epithelial cells in response to bacterial antigens, suggesting priming of innate defense pathways. Further analysis revealed differential regulation of potential epithelial cell defense genes by tears. siRNA knockdown confirmed involvement of at least two factors, RNase7 and ST-2, for which tears increased mRNA levels, in protection against bacterial invasion. Thus, the role of mucosal fluids in defense can include modulation of epithelial immunity, in addition to direct effects on microbes.
\end{abstract}

$P=$ seudomonas aeruginosa is a Gram-negative opportunistic bacterium that does not normally infect healthy epithelial-lined tissue surfaces in vivo ${ }^{1}$. However, epithelial cells derived from these tissues become exquisitely sensitive to a variety of virulence mechanisms of $P$. aeruginos $a$ and other opportunistic pathogens if grown in cell culture media in vitro ${ }^{2,3}$. One difference between the in vivo environment and standard in vitro cell culture conditions for mucosal epithelia is the presence versus absence of mucosal fluid. Indeed, it is commonly assumed that bacteriostatic or bactericidal activities of molecular factors in mucosal fluids protect against infection in vivo. Supporting a role for mucosal fluids in defense, we have previously shown that human tear fluid can protect corneal epithelial cells against $P$. aeruginosa invasion of, and cytotoxicity towards, corneal epithelial cells in vitro, and against virulence in vivo, if added with the bacterial inoculum ${ }^{4}$, 5 . However, in the same study we reported that human tear fluid removed from the eye does not consistently inhibit the growth of $P$. aeruginosa strains that it protects cells against. This result suggested mechanisms independent of direct antimicrobial activity were involved in the epithelial protective effects of tear fluid. The same is likely to be true of other mucosal fluids, many of which are known to support survival of a rich and often complex microbiota ${ }^{6-8}$.

Here, we used $P$. aeruginosa, tear fluid and corneal epithelial cells to test the hypothesis that mucosal fluid can act directly upon epithelial cells to enhance their resistance to invasion and cytotoxic virulence mechanisms of bacteria.

\section{Results}

Exposure to cells did not enhance bacteriostatic activity of tear fluid. Epithelial cell induced activation of tear fluid bacteriostatic activity would provide a different explanation for why incubation of cells with tear fluid protects the cells from $P$. aeruginosa. To explore this alternate hypothesis, bacteriostatic activity of human tears pre-exposed to cultured corneal epithelial cells overnight was compared to tear fluid that had not been preexposed to cultured cells in vitro. Results showed that three of the four P. aeruginosa strains tested could grow in human tear fluid whether or not the tears were pre-exposed to human corneal epithelial cells in vitro, with no significant increase in bacteriostatic activity noted after the tears had been exposed to cells (Fig. 1a).

Pre-exposing epithelial cells to human tear fluid increased cellular resistance to bacterial-induced invasion or cytotoxicity. We next explored the impact of human tear fluid on cellular susceptibility to $P$. aeruginosa invasion or cytotoxic virulence mechanisms. Cells pre-exposed to human tear fluid for $16 \mathrm{~h}$ were compared to cells preexposed only to media (controls) for their susceptibility to P. aeruginosa invasion and cytotoxic activity, respectively. Before adding the bacterial inoculum, tear fluid was removed by washing cells with phosphate buffered saline. After washing, cells were challenged with $10^{4} \mathrm{cfu} P$. aeruginosa for $3 \mathrm{~h}$. Cells pre-exposed to 
a

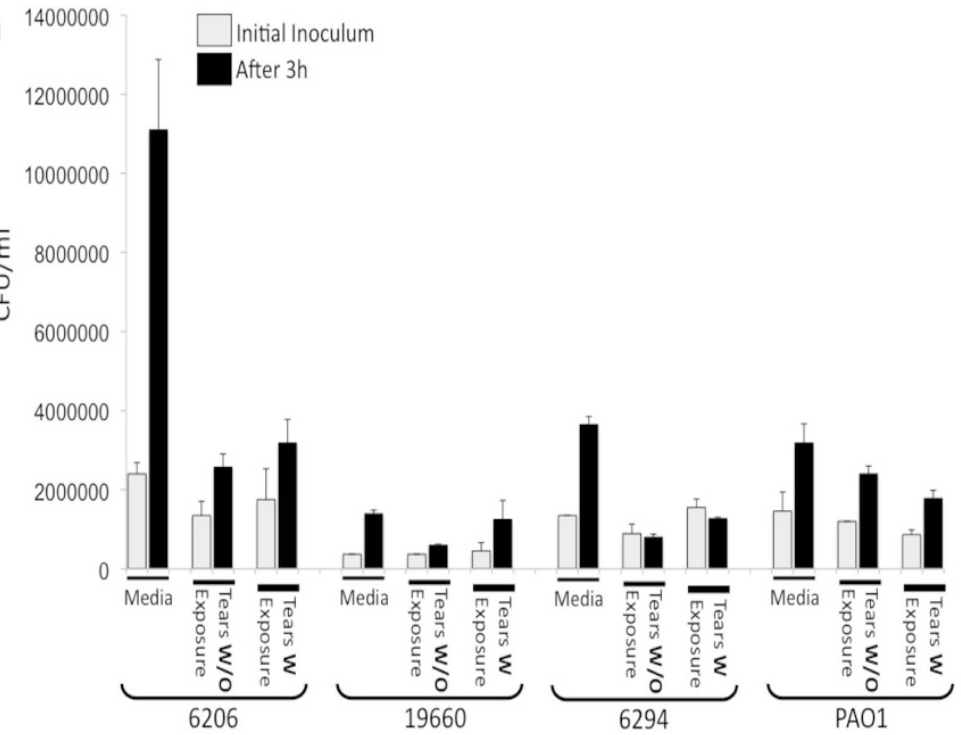

b

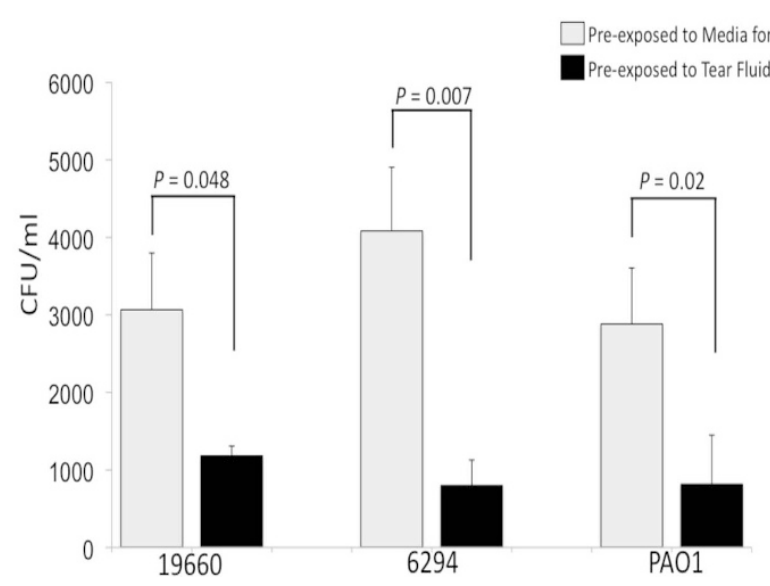

C

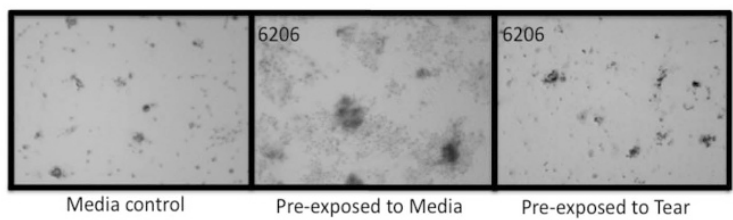

d



Figure 1 Pre-exposing Epithelial Cells to Human Tear Fluid Protects against $P$. aeruginosa Invasion and Cytotoxicity, without affecting Tear Bacteriostatic Activity. a, P. aeruginosa $\left(\sim 10^{4} \mathrm{cfu}\right)$ were suspended in cell culture media, tears, or tears pre-exposed to corneal epithelial cells for $3 \mathrm{~h}$ at $37^{\circ} \mathrm{C}$. Tear bacteriostatic activity was unaffected by prior exposure to epithelia. $\mathbf{b}, \mathbf{c}, \mathbf{d}$, . Pre-exposure to tear fluid protected corneal epithelial cells against $P$. aeruginosa invasion (b) and cytotoxicity (strain 6206) measured by trypan blue staining of dead/dying cells (c, d). Epithelial cells were exposed to tear fluid or cell culture media for $16 \mathrm{~h}$ then inoculated with $P$. aeruginosa $\left(\sim 10^{4}\right.$ cfu in cell culture media, $3 \mathrm{~h}$ at $37^{\circ} \mathrm{C}$, see methods).

tear fluid had significantly reduced susceptibility to invasion by all of three bacterial strains tested, with 2.5-, 5- and 3.5-fold reductions compared to controls observed for P. aeruginosa strains 19660, 6294 and PAO1, respectively (Fig. 1b). Tear pre-exposure also increased cellular resistance to killing by the cytotoxic $P$. aeruginosa strain 6206 $(\sim 3$ fold) (Fig. 1c, d).

Tear fluid magnified bacterial-induced activation of NFkB and AP-1 transcription factors. The above data suggested that the mechanism(s) by which tear fluid protects against $P$. aeruginosa invasion and cytotoxicity involves modulation of corneal epithelial cell defenses. Thus, we used a 10-pathway Reporter Assay, which utilizes a transcription factor-responsive firefly luciferase construct and a constitutively-expressing Renilla luciferase construct, to determine the impact of tear fluid on transcription factor activation in corneal epithelial cells in response to bacterial challenge. Corneal epithelial cells were incubated with either tears or media for $16 \mathrm{~h}$, washed, then incubated for $3 \mathrm{~h}$ with P. aeruginosa supernatant containing surface antigens (LPS, flagellin and pilin) ${ }^{9}$. Results showed that tear fluid pre-exposure magnified bacterial antigen-induced increases in luciferase expression controlled by NFאB and AP-1 transcription factors by $\sim 5-$ and 20-fold, respectively (Fig. 2a).
Impact of tear fluid on expression of specific known epithelialexpressed antimicrobial factors. Increased resistance to bacterial invasion and cytotoxicity accompanied by priming of two known innate defense signaling pathways, suggested that inducible antimicrobial factors in corneal epithelial cells might be upregulated by tear fluid. We began by exploring such factors already known to be expressed by these cells that have been shown to be inducible ${ }^{9-12}$. Thus, mRNA for hBD-2, hBD-3 and LL-37 was examined after exposure to either media or tear fluid (Fig. 2b), with or without subsequent inoculation with $P$. aeruginosa antigens (Fig. 2c). Only hBD-3 mRNA expression was increased by tear fluid, and by only $\sim 1.5$ fold (Fig. 2 b).

Since our unpublished results showed cell lysate fractions containing factors $<3 \mathrm{kDa}$ had antimicrobial activity against $P$. aeruginosa (data not shown), we next focused on molecules of that size known to be expressed by other epithelial cell types to explore if tear fluid induced their expression. Histatin 3, a precursor to histatin 5 and P113, was found expressed in significant quantities by the corneal epithelial cells, but only after tear pre-exposure (Fig. 2b). Bacterial exposure further enhanced the expression of histatin 3 in cells that had been pre-exposed to tear fluid. Histatin 5, derived by proteolytic cleavage of histatin 3, has potent antimicrobial activity and is produced by parotid and submandibular salivary glands ${ }^{13,14}$. P113 

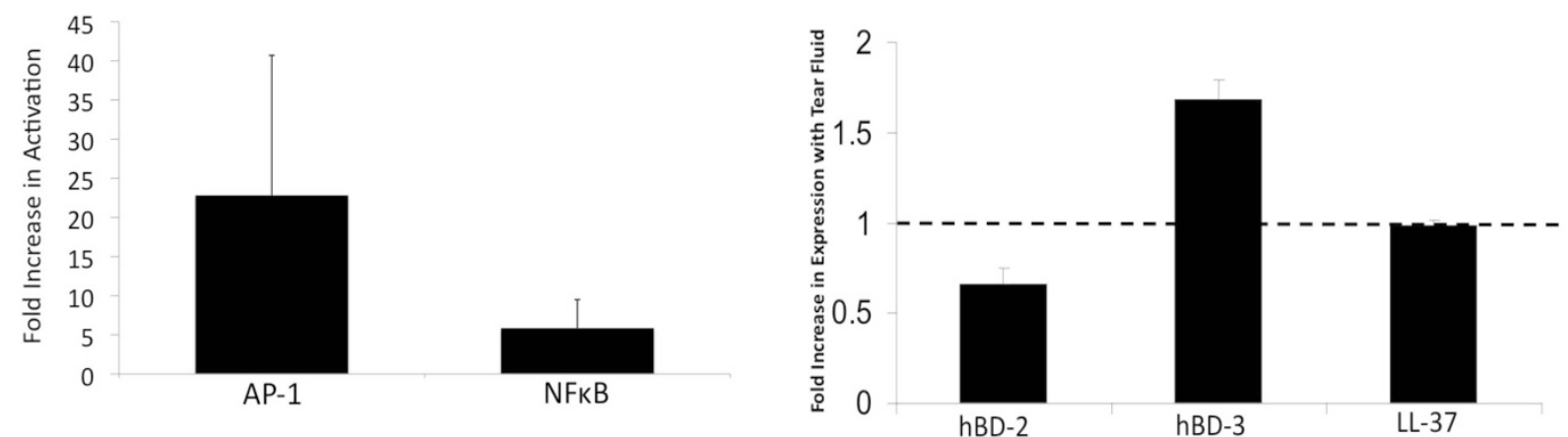

Histatin 3

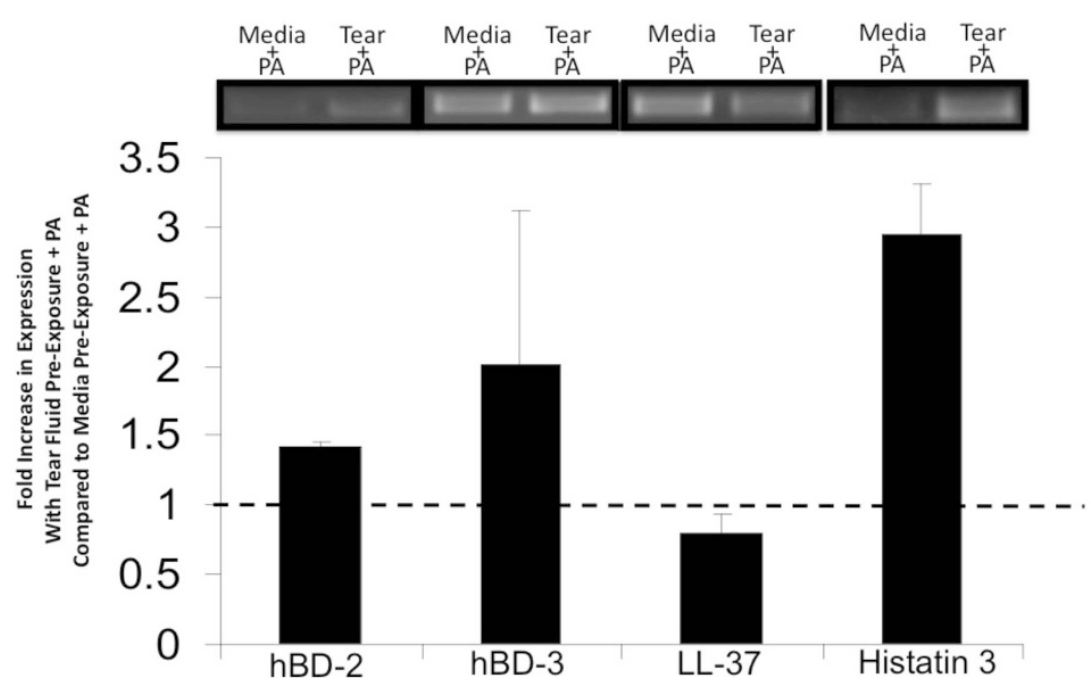

Figure 2 Tears Induce AP-1 and NFкB Activation. a, Corneal epithelial cells were transfected with NFKB or AP-1 transcription factor-responsive firefly luciferase and Renilla luciferase constructs, incubated with tears or cell culture media for $16 \mathrm{~h}$ then $3 \mathrm{~h}$ with $P$. aeruginosa antigens. Tears induced a $\sim 25$-fold increase in AP-1 activation, and $\sim 5$-fold increase in NFKB; Fold increase = cells pre-exposed to tears with $P$. aeruginosa antigens over cells preexposed to media with $P$. aeruginos a antigens b. Histatin-3 and hBD-3 mRNA induction in corneal epithelial cells exposed to tears for $6 \mathrm{~h}$ (relative to media control); c, Induction of histatin-3 mRNA in corneal cells pre-exposed to tears ( $16 \mathrm{~h}$, washed, then inoculated with $P$. aeruginos $a$ antigens for $3 \mathrm{~h}$ ).

$(<1.5 \mathrm{kDa})$, a cleaved product of its parent molecule, histatin 5 , also has potent antibacterial activity against $P$. aeruginos $a^{15}$.

Microarray analysis of tear fluid effects. To determine if there might be other critical defense genes differentially regulated by tear fluid, we performed full human genome microarray analysis of cells pre-exposed to tear fluid, with and without subsequent exposure to bacterial antigens. With exposure to tear fluid only, 192 and 29 probe sets were up- and down-regulated, respectively by at least 4 fold or more (Fig. 3a). Pre-exposure to tear fluid for cells that were then exposed to $P$. aeruginosa antigens (compared to $P$. aeruginosa antigen challenge without tear pre-exposure) up- and downregulated 151 and 160 probe sets, respectively by at least 4 fold or more (Fig. 3b). Two genes, RNase7 and ST2 were highly upregulated by multiple probe sets in both sets of experiments; tear fluid upregulated RNase7 by 28-40 fold and ST2 by 18-198 fold, and cells exposed to $P$. aeruginosa antigens with pre-exposure to tears upregulated RNase7 by $24-47$ fold and ST2 by 33-171 fold. Semiquantitative RT-PCR confirmed upregulation of these factors (Fig. 4a, b, c).

siRNA knockdown studies. Since RNase7 and ST2 have both been shown to play protective roles in immunity, and mRNA levels of each were significantly increased by tear fluid pre-exposure, which also protected cells against $P$. aeruginosa, the impact of siRNA knockdown of these molecules on epithelial cell resistance to $P$. aeruginosa was explored. Knockdown of either RNase7 or ST2, was found to significantly enhance susceptibility of the cells to $P$. aeruginosa invasion (Fig. $4 \mathrm{~d}$ ). These results suggest that each of these two factors, for which mRNA levels were increased by tear fluid pre-exposure, are directly involved in cellular resistance to $P$. aeruginosa invasion.

\section{Discussion}

The data presented in this article show that tear fluid can induce protective immunity in corneal epithelial cells against $P$. aeruginosa invasion and cytotoxic virulence mechanisms. Tear fluid exposure was found to change the expression of many genes, with the most significant increased mRNA levels observed for RNase7 and ST2. Accordingly, knockdown of either RNase7 or ST2 increased cellular susceptibility to $P$. aeruginosa invasion, showing both factors play a role in tear fluid-induced epithelial immunity to this pathogen.

RNase7 is a known antimicrobial $(\sim 14.5 \mathrm{kDa})$ with potent ribonuclease activity first identified in human skin, found to play a major role in determining the skin antimicrobial barrier ${ }^{16-18}$. It has potent antimicrobial activity against various pathogens including E. coli, Pseudomonas aeruginosa, Candida albicans, Staphylococcus 
a

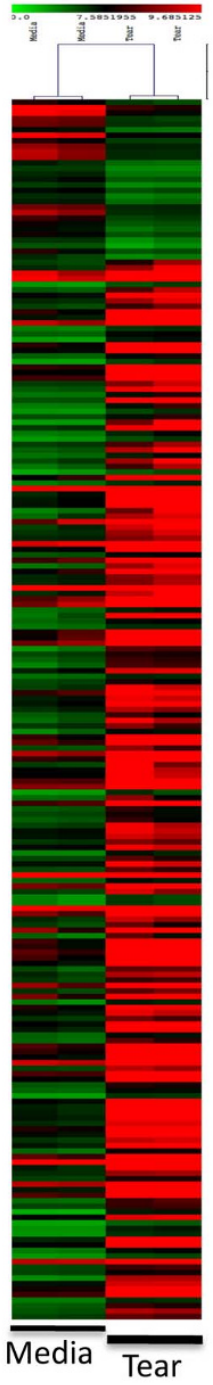



b

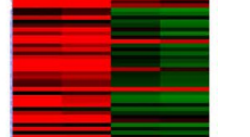

also explain its capacity to reduce pathology during infection; i.e., modulation of epithelial cell susceptibility to bacterial virulence factors. Given the complexity of already known effects of ST2, particularly on TLR signaling ${ }^{23,29}$, further studies will be needed to elucidate exactly how ST2 protects cells against bacteria. However, possibilities include that it impacts production of epithelial-expressed antimicrobials via its effects on TLR-mediating signaling or Th2 responses, which each regulate expression of antimicrobial peptides ${ }^{30,}{ }^{31}$. Alternatively ST2 could impact, via effects on microbe recognition, bacterial trafficking to intraepithelial perinuclear vacuoles that are capable of killing intracellular bacteria ${ }^{32}$. Tear fluid was found to prime cells for NFKB and AP-1 transcription factor activation, supporting that downstream innate immune responses are impacted.

Recent studies have shown that the normal microbiota at some mucosal surfaces can induce immunity in epithelial cells lining the mucosa $^{6,33,34}$ involving TLRs $^{35}$ which detect microbe-associated molecular patterns (MAMPs) ${ }^{36}$. DAMPs (damage associated molecular patterns) can also trigger TLR receptor signaling and therefore downstream effects ${ }^{37}$. Since the ocular surface lacks significant microbiota, other mechanisms must be involved in maintaining the normal immune tone of its surface epithelial cells during health. Data presented in this report showing that tear fluid can modulate epithelial immunity suggests the presence of TAMPs (tear associated molecular patterns). Whether these act on the same receptors/signaling pathways as other factors that modulate epithelial immunity, and whether components of tears responsible are similar in structure to MAMPs or PAMPs, remains to be determined.

While we have found changes to gene expression induced by tear fluid, some involved in epithelial immunity to bacteria, it remains possible that tear fluid influences cell immunity in other ways, for example if tear components directly impact the surface biochemistry of the epithelial cells, e.g. by binding directly to cells or by modifying cellular factors directly. Further studies will be needed to address that possibility.

The significance of these findings extends to the design of in vitro cell culture studies. Standard methods for studying epithelial cell biology, including cellular microbiology, involve growth of cells in tissue culture media. The results of this study suggest that growth media should be formulated to mimic the appropriate mucosal fluid when studying mucosal epithelial cells in vitro.

Factors that stagnate mucosal fluids often cause susceptibility to infection, such as cystic fibrosis, intubation, and contact lens wear. While reduced clearance of microbes and/or biofilm formation very likely contributes to susceptibility in these instances, the impact of prolonged stagnation on mucosal fluid biochemistry and its subsequent impact on epithelial immune tone, could be another topic worthy of investigation.

In summary, the results of this study show that the role of mucosal fluids in defense against infection stretches beyond effects on microbe viability or virulence to actually enhancing cellular resistance to microbes. These findings add to our understanding of how mucosal surfaces resist infection during health, and suggest new avenues for developing biocompatible agents to prevent, rather than simply treat, pathology in susceptible patients.

\section{Methods}

Cell culture. Human telomerase-immortalized corneal epithelial cells (hTCEpi) were maintained in $10 \mathrm{~cm}$ tissue culture treated Petri dishes (Becton Dickinson, Franklin Lake, NJ) in serum-free KGM-2 medium (Lonza, Walkersville, MD) until confluent as previously described ${ }^{38}$. Cells were then seeded onto 96 -well tissue culture plate (Becton Dickinson, Franklin Lake, NJ) and grown to $\sim 80-90 \%$ confluence. 2 days prior to experiment, the cells were incubated in high calcium KGM-2 medium containing $1.15 \mathrm{mM} \mathrm{Ca}^{2+}$ for $16 \mathrm{~h}$, then washed with sterile phosphate-buffered saline (PBS; Sigma, St. Louis, MO) before being incubated in either $40 \mu \mathrm{l}$ of fresh human tear fluid or high calcium KGM-2 without antibiotics either for $6 \mathrm{~h}$ or $16 \mathrm{~h}$ depending on the experiment. All cells were incubated at $37^{\circ} \mathrm{C}$ with $5 \% \mathrm{CO}_{2}$ while culturing and during experiments. 
a

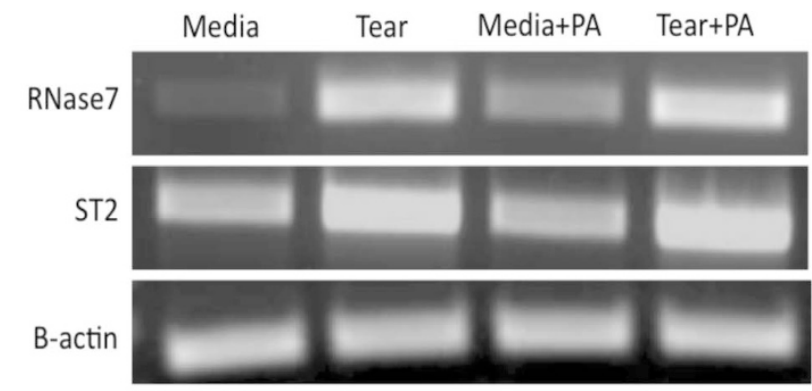

b

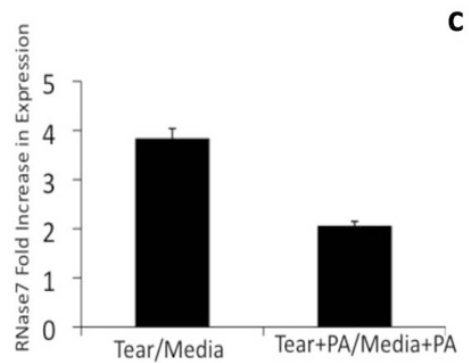

C

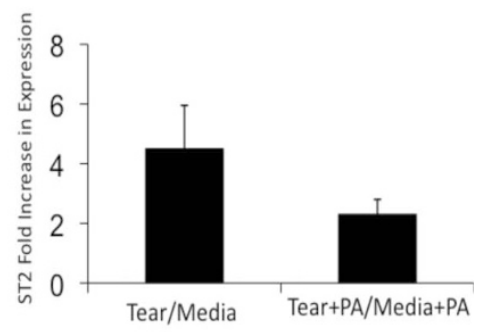

d

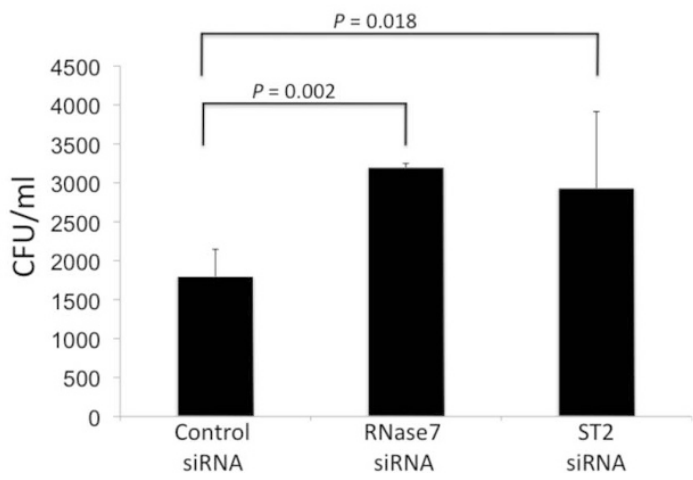

Figure 4 RT-PCR Confirmation of Tear Induced Expression of RNase7 and ST2 and Their Protective Effect against $P$. aeruginosa Invasion. a, b, c, Semi-quantitative RT-PCR confirmed that $6 \mathrm{~h}$ tear exposure alone or $16 \mathrm{~h}$ pre-exposure to tear fluid followed by $3 \mathrm{~h}$ incubation with $P$. aeruginosa antigens induced RNase7 and ST2 mRNA expression in corneal epithelial cells. d, siRNA knockdown of RNase7 or ST2 in corneal epithelial cells increased $P$. aeruginosa invasion.

Preparation of bacteria. All bacteria were grown on trypticase soy agar plates overnight at $37^{\circ} \mathrm{C}$. Bacteria were suspended in basal KBM-2 media (without antibiotics) to a spectrophotometer optical density of 0.1 at $\mathrm{OD}_{650}$ which is equivalent to $\sim 1 \times 10^{8} \mathrm{cfu} / \mathrm{mL}$ and diluted to $\sim 1 \times 10^{6} \mathrm{cfu} / \mathrm{mL}$ for invasion and cytotoxicity experiments. P. aeruginosa clinical isolates 6294 and 6206 were used for invasion and cytotoxicity assays, respectively. Some experiments also involved the use of two laboratory isolates of P. aeruginosa, strains PAO1 and 19960.

P. aeruginosa supernatant. $P$. aeruginosa strain PAO1 was grown as single colonies on trypticase soy agar at $37^{\circ} \mathrm{C}$ overnight. A single colony was then inoculated into $5 \mathrm{ml}$ of trypticase soy broth and grown with aeration to late log phase $\left(37^{\circ} \mathrm{C}\right)$. The culture broth was then centrifuged at $14,000 \mathrm{rpm}(16,000 \times \mathrm{g})$ for $30 \mathrm{~min}$ and the supernatant collected in a syringe, sterilized with a $0.22 \mu \mathrm{m}$ polymer filter (Corning Star Corporation, Cambridge, MA), aliquoted and stored at $-80{ }^{\circ} \mathrm{C}$ until use in experiments. Bacterial supernatant diluted 1:5 with basal KBM-2 media (without antibiotics), or an equivalent amount of basal cell culture medium, was used to stimulate corneal epithelial cells for experiments.

Invasion and cytotoxicity assays. Corneal epithelial cells were either pre-exposed to high $\mathrm{Ca}^{2+}$ media or human tear fluid for $16 \mathrm{~h}$ (for tear fluid pre-exposure experiments), washed with $100 \mu \mathrm{l}$ of pre-warmed PBS, then inoculated with $40 \mu \mathrm{l}$ of $P$. aeruginosa strain 6294 or strain 6206 diluted in basal KBM-2 media to a concentration of $\sim 1 \times 10^{6} \mathrm{CFU} / \mathrm{ml}$. Triplicate wells were used for each sample. Epithelial cells were incubated with bacteria for $3 \mathrm{~h}\left(37^{\circ} \mathrm{C}, 5.0 \% \mathrm{CO}_{2}\right)$. The cells were then washed with PBS and incubated with $100 \mu \mathrm{l}$ of gentamicin solution $(0.4 \%$ [vol/vol]; $200 \mu \mathrm{g} / \mathrm{ml}$; BioWhittaker, Walkersville, MD) in cell culture media. After a $1 \mathrm{~h}$ incubation at $37^{\circ} \mathrm{C}, 100 \mu \mathrm{l}$ of Trypan blue solution $(10 \%[\mathrm{vol} / \mathrm{vol}])$ in basal KBM-2 media was added, and incubated for $15 \mathrm{~min}$. The Trypan blue solution was then replaced with $100 \mu \mathrm{l}$ of basal KBM-2 media. Phase-contrast images were taken on an inverted microscope (Olympus IX-70, Olympus America Inc., Center Valley, PA) attached to a video camera (Optronics, Goleta, CA) at $200 \times$ magnification. Epithelial cells were considered vulnerable to bacterial cytotoxicity (i.e. dead/dying) if they exhibited Trypan blue staining. Blue stained cells were also quantified for statistical analysis. To quantify intracellular bacteria, epithelial cells were lysed in Triton X-100 (0.25\% [vol/vol]; LabChem Inc., Pittsburgh, PA) in PBS for 15 minutes. The cells were then scraped for complete lysis and undiluted and $10^{-1}$ dilutions plated onto MacConkey agar plates to enumerate surviving (intracellular) bacteria by viable count.

Bacterial growth assay. Bacterial growth and viability were tested by adding $50 \mu \mathrm{l}$ of bacteria suspended in either cell culture media, or tears with or without exposure to corneal cells, to empty Eppendorf tubes. These suspensions were incubated at $37^{\circ} \mathrm{C}$ for $3 \mathrm{~h}$ and quantified by viable count. Initial suspensions were also quantified to study bacteriostatic or bactericidal effects of tear fluid. These were done in triplicates.

Transfection and luciferase array assay. Human corneal epithelial cells were reverse transfected using SureFECT (SABiosciences, Frederick, MD) onto Cancer 10-Pathway Reporter Array (SABiosciences, Frederick, MD). Each reporter consists of a mixture of an inducible transcription factor responsive firefly luciferase reporter and a constitutively expressing Renilla luciferase construct $(20: 1)$. Firefly and Renilla luciferase activities of cells incubated either in media or tear fluid for $16 \mathrm{~h}$ followed by 3 h incubation with $P$. aeruginosa supernatant were measured. The change in the activity of each signaling pathway was calculated by comparing the normalized luciferase activities of the reporter of each group.

RNA purification. Total RNA of corneal epithelial cells incubated in either cell culture media or human tear fluid for $6 \mathrm{~h}$ and cells either pre-exposed to media or tear fluid for $16 \mathrm{~h}$ followed by $3 \mathrm{~h}$ incubation with $P$. aeruginosa supernatant were 
extracted with the RNeasy Kit (Qiagen, Valencia, CA) using Qiashredder columns for cell lysis and inserting Qiagen on-column DNase steps to remove any contaminating genomic DNA.

RNA target preparation/GeneChip microarray analysis. RNA quality was assessed by using the Agilent Model 2100 Bioanalyzer (Agilent Technologies, Palo Alto, CA). Ten micrograms of total RNA was processed for use on the microarray by using the Affymetrix GeneChip one-cycle target labeling kit (Affymetrix, Santa Clara, CA) according to the manufacturer's recommended protocols. The resultant biotinylated cRNA was fragmented and then hybridized to the GeneChip Human Genome U133 Plus 2.0 Arrays (Affymetrix). The arrays were washed, stained, and scanned using the Affymetrix Model 450 Fluidics Station and Affymetrix Model 3000 scanner, following manufacturer's recommended protocols, at the University of California Functional Genomics Laboratory. Expression values were generated by using Microarray Suite (MAS) v5.0 software (Affymetrix). Each sample and hybridization underwent a quality control evaluation, including cRNA amplification of $>4$-fold, percentage of probe sets reliably detecting between $40 \%$ and $60 \%$ present call, and $3^{\prime}-5^{\prime}$ ratio of GAPDH gene.

\section{Evaluation and normalization of affymetrix GeneChip data. The hybridizations} were normalized by using the RMA (robust multichip averaging) method from Bioconductor to obtain summary expression values for each probe set. Gene expression levels were analyzed on a logarithmic scale. The given backgroundcorrected and normalized $\log _{2}$ values were anti-logged and fold expression level changes were determined by dividing the experimental group by the control. Any gene showing differential expression by at least four fold and $p=0.01$ was subjected to Affymetrix Annotation Files. Functional gene classifications were derived from Gene Ontology information found in National Center for Biotechnology Information (NCBI) database, Onto-tools (Intelligent Systems and Bioinformatics Laboratory website, (http://vortex.cs.wayne.edu/Projects.html) and from independent literature searches. The heat-map was generated using MeV.

Semi-quantitative RT-PCR. $1 \mu \mathrm{g}$ of total purified RNA was converted to cDNA using RETROscript Kit (Ambion, Austin, Texas). The expression of hBD-2, -3, LL-37, histatin 3, ST2 and RNase7 mRNAs were assessed for each condition mentioned previously using intron spanning primers. As an internal loading control, primers specific for B-actin was used. Polymerase chain reaction (PCR) products were separated on $2 \%$ agarose gel and visualized by ethidium bromide. Band intensities were quantified using the FluorChem Q SA software (Alpha Innotech, Santa Clara, $\mathrm{CA}$ ) and normalized with those of $\beta$-actin and medium treated controls. Experiments were repeated at least three times.

siRNA. HCECs were transfected with control siRNA ( $50 \mathrm{nmol} / \mathrm{L})$, ST2 siRNA $(50 \mathrm{nmol} / \mathrm{L})$, and RNase7 siRNA ( $50 \mathrm{nmol} / \mathrm{L})$ by the use of Lipofectamine RNAi Max (Invitrogen, Carlsbad, CA) for 6 hours. Cells were used for assays at 48 hours after transfection. Expression levels of RNase7 and ST2 were determined by semiquantitative RT-PCR. Small interfering (si)RNAs were designed by Dharmacon Inc (Lafayette, CO)

Statistical analysis. Data were expressed as mean $\pm s . d$ ( $n=3$ or 4 samples per group) unless otherwise stated. Student's t-test was used to evaluate the statistical significance of differences between groups with $\mathrm{P}$ values $<0.05$ considered significant. All experiments were repeated at least three times.

1. Mun, J. J., Tam, C., Kowbel, D., Hawgood, S., Barnett, M. J., Evans, D. J. \& Fleiszig, S. M. Clearance of Pseudomonas aeruginosa from a healthy ocular surface involves surfactant protein $\mathrm{D}$ and is compromised by bacterial elastase in a murine nullinfection model. Infect Immun. 77, 2392-8 (2009).

2. Fleiszig, S. M., Lee, E. J., Wu, C., Andika, R. C., Vallas, V., Portoles, M. \& Frank, D. W. Cytotoxic strains of Pseudomonas aeruginosa can damage the intact corneal surface in vitro. CLAO J. 24, 41-7 (1998).

3. Fleiszig, S. M., Zaidi, T. S. \& Pier, G. B. Pseudomonas aeruginosa invasion of and multiplication within corneal epithelial cells in vitro. Infect Immun. 63, 4072-7 (1995)

4. Fleiszig, S. M., Kwong, M. S. \& Evans, D. J. Modification of Pseudomonas aeruginosa interactions with corneal epithelial cells by human tear fluid. Infect Immun. 71, 3866-74 (2003).

5. Kwong, M. S., Evans, D. J., Ni, M., Cowell, B. A. \& Fleiszig, S. M. Human tear fluid protects against Pseudomonas aeruginosa keratitis in a murine experimental model. Infect Immun. 75, 2325-32 (2007).

6. Didierlaurent, A., Simonet, M. \& Sirard, J. C. Innate and acquired plasticity of the intestinal immune system. Cell Mol Life Sci. 62, 1285-7 (2005).

7. Hooper, L. V. \& Gordon, J. I. Commensal host-bacterial relationships in the gut. Science. 292, 1115-8 (2001).

8. Reid, G., Younes, J. A., Van der Mei, H. C., Gloor, G. B., Knight, R. \& Busscher, H. J. Microbiota restoration: natural and supplemented recovery of human microbial communities. Nat Rev Microbiol. (2010).

9. Mc, N. N., Van, R., Tuchin, O. S. \& Fleiszig, S. M. Ocular surface epithelia express mRNA for human beta defensin-2. Exp Eye Res. 69, 483-90 (1999).

10. Gordon, Y. J., Huang, L. C., Romanowski, E. G., Yates, K. A., Proske, R. J. \& McDermott, A. M. Human cathelicidin (LL-37), a multifunctional peptide, is expressed by ocular surface epithelia and has potent antibacterial and antiviral activity. Curr Eye Res. 30, 385-94 (2005).

11. Harder, J., Bartels, J., Christophers, E. \& Schroder, J. M. Isolation and characterization of human beta -defensin-3, a novel human inducible peptide antibiotic. J Biol Chem. 276, 5707-13 (2001).

12. Lehrer, R. I. \& Ganz, T. Cathelicidins: a family of endogenous antimicrobial peptides. Curr Opin Hematol. 9, 18-22 (2002).

13. Edgerton, M. \& Koshlukova, S. E. Salivary histatin 5 and its similarities to the other antimicrobial proteins in human saliva. Adv Dent Res. 14, 16-21 (2000).

14. Kavanagh, K. \& Dowd, S. Histatins: antimicrobial peptides with therapeutic potential. J Pharm Pharmacol. 56, 285-9 (2004).

15. Sajjan, U. S., Tran, L. T., Sole, N., Rovaldi, C., Akiyama, A., Friden, P. M., Forstner, J. F. \& Rothstein, D. M. P-113D, an antimicrobial peptide active against Pseudomonas aeruginosa, retains activity in the presence of sputum from cystic fibrosis patients. Antimicrob Agents Chemother. 45, 3437-44 (2001).

16. Harder, J. \& Schroder, J. M. RNase 7, a novel innate immune defense antimicrobial protein of healthy human skin. J Biol Chem. 277, 46779-84 (2002).

17. Zasloff, M. Antimicrobial RNases of human skin. J Invest Dermatol. 129, 2091-3 (2009).

18. Zhang, J., Dyer, K. D. \& Rosenberg, H. F. Human RNase 7: a new cationic ribonuclease of the RNase A superfamily. Nucleic Acids Res. 31, 602-7 (2003).

19. Lin, Y. M., Wu, S. J., Chang, T. W., Wang, C. F., Suen, C. S., Hwang, M. J., Chang, M. D., Chen, Y. T. \& Liao, Y. D. Outer membrane protein I of Pseudomonas aeruginosa is a target of cationic antimicrobial peptide/protein. J Biol Chem. 285, 8985-94 (2010)

20. Otri, A. M., Mohammed, I., Abedin, A., Cao, Z., Hopkinson, A., Panjwani, N. \& Dua, H. S. Antimicrobial peptides expression by ocular surface cells in response to Acanthamoeba castellanii: an in vitro study. Br J Ophthalmol. 94, 1523-7 (2010).

21. Yanagisawa, K., Naito, Y., Kuroiwa, K., Arai, T., Furukawa, Y., Tomizuka, H. Miura, Y., Kasahara, T., Tetsuka, T. \& Tominaga, S. The expression of ST2 gene in helper T cells and the binding of ST2 protein to myeloma-derived RPMI8226 cells. J Biochem. 121, 95-103 (1997).

22. Trajkovic, V., Sweet, M. J. \& Xu, D. T1/ST2-an IL-1 receptor-like modulator of immune responses. Cytokine Growth Factor Rev. 15, 87-95 (2004).

23. Li, X. \& Qin, J. Modulation of Toll-interleukin 1 receptor mediated signaling. J Mol Med. 83, 258-66 (2005)

24. Humphreys, N. E., Xu, D., Hepworth, M. R., Liew, F. Y. \& Grencis, R. K. IL-33, a potent inducer of adaptive immunity to intestinal nematodes. J Immunol. 180, 2443-9 (2008)

25. Walzl, G., Matthews, S., Kendall, S., Gutierrez-Ramos, J. C., Coyle, A. J., Openshaw, P. J. \& Hussell, T. Inhibition of T1/ST2 during respiratory syncytial virus infection prevents Thelper cell type 2 (Th2)- but not Th1-driven immunopathology. J Exp Med. 193, 785-92 (2001).

26. Xu, D., Chan, W. L., Leung, B. P., Hunter, D., Schulz, K., Carter, R. W., McInnes, I. B., Robinson, J. H. \& Liew, F. Y. Selective expression and functions of interleukin 18 receptor on Thelper (Th) type 1 but not Th2 cells. J Exp Med. 188, 1485-92 (1998).

27. Huang, X., Du, W., Barrett, R. P. \& Hazlett, L. D. ST2 is essential for Th2 responsiveness and resistance to Pseudomonas aeruginosa keratitis. Invest Ophthalmol Vis Sci. 48, 4626-33 (2007).

28. Hazlett, L. D., McClellan, S. A., Barrett, R. P., Huang, X., Zhang, Y., Wu, M., van Rooijen, N. \& Szliter, E. IL-33 shifts macrophage polarization, promoting resistance against Pseudomonas aeruginosa keratitis. Invest Ophthalmol Vis Sci. 51, 1524-32 (2010)

29. Kakkar, R. \& Lee, R. T. The IL-33/ST2 pathway: therapeutic target and novel biomarker. Nat Rev Drug Discov. 7, 827-40 (2008).

30. O’Neil, D. A., Porter, E. M., Elewaut, D., Anderson, G. M., Eckmann, L., Ganz, T. \& Kagnoff, M. F. Expression and regulation of the human beta-defensins hBD-1 and hBD-2 in intestinal epithelium. J Immunol. 163, 6718-24 (1999).

31. Wang, X., Zhang, Z., Louboutin, J. P., Moser, C., Weiner, D. J. \& Wilson, J. M. Airway epithelia regulate expression of human beta-defensin 2 through Toll-like receptor 2. FASEB J. 17, 1727-9 (2003).

32. Angus, A. A., Lee, A. A., Augustin, D. K., Lee, E. J., Evans, D. J. \& Fleiszig, S. M. Pseudomonas aeruginosa induces membrane blebs in epithelial cells, which are utilized as a niche for intracellular replication and motility. Infect Immun. 76, 1992-2001 (2008).

33. Endt, K., Stecher, B., Chaffron, S., Slack, E., Tchitchek, N., Benecke, A., Van Maele, L., Sirard, J. C., Mueller, A. J., Heikenwalder, M., Macpherson, A. J., Strugnell, R., von Mering, C. \& Hardt, W. D. The microbiota mediates pathogen clearance from the gut lumen after non-typhoidal Salmonella diarrhea. PLoS Pathog. 6, (2010).

34. O’Mahony, C., Scully, P., O’Mahony, D., Murphy, S., O’Brien, F., Lyons, A., Sherlock, G., MacSharry, J., Kiely, B., Shanahan, F. \& O’Mahony, L. Commensalinduced regulatory $\mathrm{T}$ cells mediate protection against pathogen-stimulated NFkappaB activation. PLoS Pathog. 4, e1000112 (2008).

35. Rakoff-Nahoum, S., Paglino, J., Eslami-Varzaneh, F., Edberg, S. \& Medzhitov, R. Recognition of commensal microflora by toll-like receptors is required for intestinal homeostasis. Cell. 118, 229-41 (2004). 
36. Ausubel, F. M. Are innate immune signaling pathways in plants and animals conserved? Nat Immunol. 6, 973-9 (2005).

37. Piccinini, A. M. \& Midwood, K. S. DAMPening inflammation by modulating TLR signalling. Mediators Inflamm. 2010, (2010).

38. Robertson, D. M., Li, L., Fisher, S., Pearce, V. P., Shay, J. W., Wright, W. E., Cavanagh, H. D. \& Jester, J. V. Characterization of growth and differentiation in a telomerase-immortalized human corneal epithelial cell line. Invest Ophthalmol Vis Sci. 46, 470-8 (2005).

\section{Acknowledgements}

This work was supported by a Grand Challenges Explorations (GCE) Grant from the Bill and Melinda Gates Foundation (SMJF and DJE), and by NIH research grant R01-EY011221 (SMJF).

\section{Author contributions}

$\mathrm{JM}$ and $\mathrm{CT}$ performed all experimental work. All authors contributed to the experimental design, interpretation of data, and writing the manuscript. SMJF and DJE supervised the project.

\section{Additional information}

Competing financial interests: The authors declare no competing financial interests.

License: This work is licensed under a Creative Commons

Attribution-NonCommercial-ShareAlike 3.0 Unported License. To view a copy of this license, visit http://creativecommons.org/licenses/by-nc-sa/3.0/

How to cite this article: Mun, J. J., Tam, C. K., Evans, D. J. \& Fleiszig, S. M. J. Modulation of Epithelial Immunity by Mucosal Fluid. Sci. Rep. 1, 8; DOI:10.1038/srep00008 (2011). 